\title{
Dialect Identification versus Evaluation of Risk in Language Screening
}

\author{
Lois Ciolli, M.A., C.C.C.-S.L.P., ${ }^{1}$ and Harry N. Seymour, Ph.D. ${ }^{2}$
}

This article proposes that any dialect-neutral screening test should consist of two parts: one part using contrastive items to screen for Language Variation Status (Mainstream American English [MAE] or a degree of variation from MAE), and a second part using noncontrastive items to screen for degree of risk for language disorder (low, medium, or high). The two scores are interpreted together in forming an overall clinical profile of a child. The implications for further diagnostic testing of both MAE and non-MAE speakers who exhibit risk for language disorder are discussed. Most importantly, for the African American child, viewing the results of the two types of proposed screening items together clears the "variation due to speech and language dialect" issue out of the way so that if further evaluation is needed, it is done for valid reasons, not superficial ones.

KEYWORDS: Language Variation Status, Diagnostic Risk Status, degree of risk, variation from mainstream, optimal, obligatory, contrastive aspects of language, noncontrastive aspects of language, risk for disorder

\begin{abstract}
Learning Outcomes: As a result of this activity, the participant will be able to (1) describe contrastive aspects of language that can be used to identify African American English (AAE) dialect usage in children ages 4 to 12 years, and (2) describe noncontrastive aspects of language that can be used to determine degree of risk for a language disorder in children ages 4 to 9 years, regardless of whether AAE or Mainstream American English (MAE) is spoken.
\end{abstract}

\section{DIALECT IDENTIFICATION VERSUS RISK DIAGNOSIS}

If the question for a screening procedure is to identify dialect versus disorder in African
American English (AAE) speakers, both goals can be accomplished with phonology and morphosyntax items. For example, in phonology, consonant clusters when in word-final position

\footnotetext{
Evaluating Language Variation: Distinguishing Dialect and Development from Disorder; Editors in Chief, Nancy HelmEstabrooks, Sc.D., and Nan Bernstein Ratner, Ed.D.; Guest Editors, Harry N. Seymour, Ph.D., and Barbara Zurer Pearson, Ph.D. Seminars in Speech and Language, volume 25, number 1, 2004. Address for correspondence and reprint requests: Lois Ciolli, M.A., C.C.C.-S.L.P., The Psychological Corporation, Harcourt Assessment, Inc., 19500 Bulverde Road, San Antonio TX 78259. E-mail: lois_ciolli@harcourt.com. ${ }^{1}$ Senior Research Director, The Psychological Corporation, Harcourt Assessment, Inc., San Antonio, Texas; ${ }^{2}$ Professor Emeritus, Department of Communication Disorders, University of Massachusetts Amherst, Amherst, Massachusetts. Copyright (C) 2004 by Thieme Medical Publishers, Inc., 333 Seventh Avenue, New York, NY 10001, USA. Tel: +1(212) 584-4662. 0734-0478,p;2004,25,01, 033,040,ftx,en;ss100181x.
} 
have different rules in AAE and MAE. Therefore, if the purpose is to establish Language Variation Status, the phonology items should target only the final position, where differences between $\mathrm{AAE}$ and $\mathrm{MAE}$ are greatest. In contrast, in screening for phonological disorder, it is reasonable to present targets only in initial and medial positions, where AAE and MAE speakers (for the most part) treat them alike.

Similarly, in morphosyntax, the distinct sets of dialect identifier and language disorder screening items can both include items involving the past tense verb was (or were). For the identifier (Language Variation Status) objective, the focus of these items should be the nature of the subject-verb agreement, which differs reliably across the dialects: in AAE, was occurs predictably with plural they ("they was"), whereas MAE speakers almost exclusively say "they were." In contrast, the items in a screener for language disorder should focus on whether was is deleted or not; neither dialect would permit deletion in that context. ${ }^{1,2}$ In the present tense, AAE forms of to be are used optionally, ${ }^{3}$ but past tense was or were are obligatory in AAE because they carry the tense marking, information essential to understanding the sentence. If a child of either dialect deletes was, that would be a marker of disorder. Thus, the contrastive agreement pattern for was can be used in determining Language Variation Status, but the noncontrastive obligatory presence of was can be used for determining risk status.

\section{Item Goals}

\section{PART I: DEVELOPING A LANGUAGE VARIATION SCREENER}

We constructed a potential set of items with the goal to maximize the difference in responses between the AAE and MAE speakers. Given that the most obvious contrastive features between the two dialects are morphological and phonological, those features were the focus of research for this part of the battery we developed. Out of 182 morphosyntax and phonology items that were tested during the preliminary research, 15 items that best differentiated the two dialect groups were selected for the final version of our proposed screening test. Although there are many other features typically considered characteristic of AAE, they are not represented in our final version (e.g., multiple negation, "he don't have no shoes"). The items selected were those that best differentiated the two dialect groups in morphological and phonological patterns at all of the ages we looked at. The end result was 5 phonology and 10 morphosyntax items that seemed quick to administer and score.

We found that the most discriminating phonology items involved only three phonemes - voiceless " $\theta$," voiced " $\partial$," and the consonant cluster $/ \mathrm{ft} /$. These were incorporated into five phonology items as stimulus targets and were designed to elicit AAE patterns in the final position of words: $/ \theta / \longrightarrow[\mathrm{f}]$; /ð/ $\rightarrow[\mathrm{v}] ; /$ / $\rightarrow[\mathrm{d}] ; /$ / $\rightarrow$ zero; and $/ \mathrm{ft} / \rightarrow[\mathrm{f}]$. In administering these five phonological targets, the examiner says a short sentence about the picture and asks the child to repeat it. For example, the examiner might show the child a picture of a child's face with her mouth open and say, "I see she can open her mouth."

The next 10 items that we field tested focus on morphosyntactic aspects of the child's speech. For these items, the child is asked to look at a picture and finish a sentence or answer a question about it. The items assess a child's marking of third-person, present tense, singular verbs (has, -s, -es, does) and the third-person, past tense, plural verb agreement, as in "they was" or "they were."

A child's dialect patterns can be easily identified with this set of proposed items. We have found that a child who is a speaker of AAE will respond to most of the 15 target items in a manner typical of AAE. Likewise, in response to the same 15 items, a child who is a speaker of MAE will produce more MAE patterns. Nonresponses or response patterns not typical of either the predicted AAE or MAE targets also could be recorded, but not entered into the scoring. The combined score for the phonology and morphology items can be used to determine a child's variation status as either "MAE," "Some Variation from MAE," or "Strong Variation from MAE." As seen in 


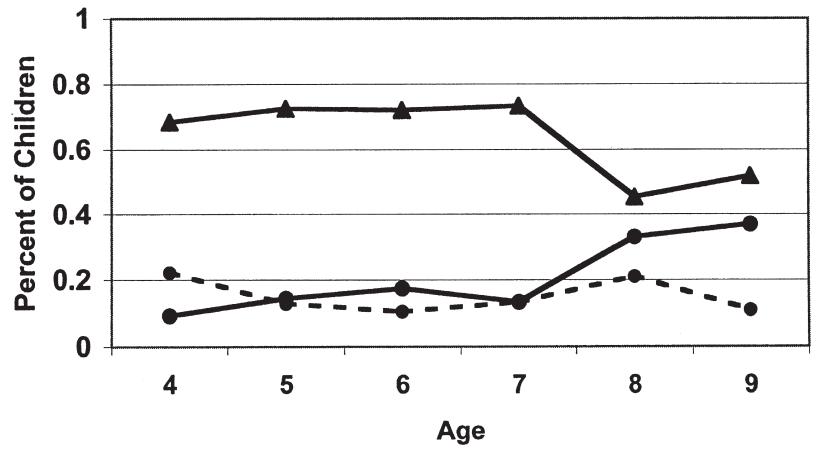

$\longrightarrow$ MAE - $\rightarrow-$-Some Variation $\longrightarrow$ Strong Variation

Figure 1 Language variation categories (African American English [AAE]-background children). MAE, Mainstream American English.

Figures 1 and 2, our experimental work shows that the dialect groups have very different numbers of children in each of these categories.

Typically developing children from MAE family backgrounds rarely show any Language Variation Status except MAE (Fig. 2), whereas children from AAE family backgrounds show all three patterns (Fig. 1). Even at the older ages when many of the children are learning to codeswitch into MAE, typically developing African American children are still more likely to speak with some or strong variation from MAE.

\section{Item Goals-Part II: Diagnostic Risk Status}

We next sought items that were effective in identifying children at risk for a language disorder and in eliciting the same responses from typically developing children, regardless of whether they were AAE or MAE speakers. Out of the 313 items that were researched in the second phase of piloting, we found 17 items that as a group best discriminated between the typically developing children and those with language impairments in the research sample.

Five of the items focus on the child's production of the third-person singular past tense verb was, in either copula or auxiliary form. Two more of the items that we identified for this function also focus on morphosyntax; these are possessive pronouns. Four more items involve $w h$-questions and are discussed by Roeper. ${ }^{4}$ Again, both the typically developing $\mathrm{AAE}$ and MAE speakers understood these in the same manner.

The last set of items that we developed arose from work done by Campbell et $\mathrm{al}^{5}$ on nonword repetition abilities of children with and without specific language impairment

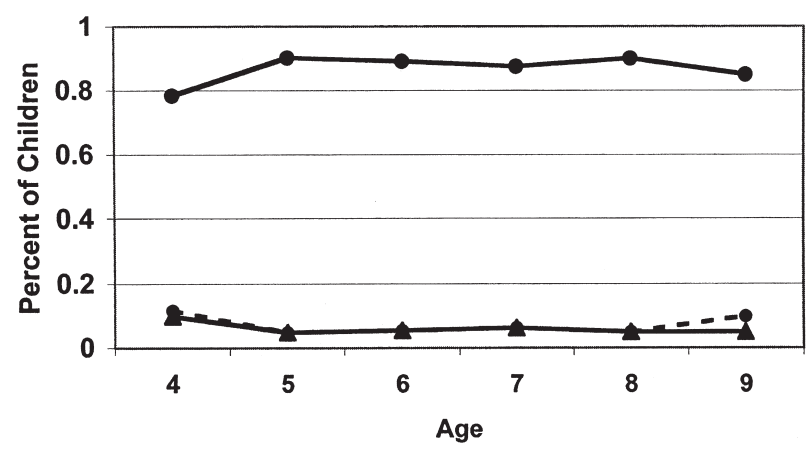

$\longrightarrow$ MAE - $\rightarrow--$ Some Variation $\longrightarrow-$ Strong Variation

Figure 2 Language variation categories (Mainstream American English [MAE]-background children). 
(SLI). Nonword repetition tasks have shown to be useful in the identification of SLI children without biasing effects toward speakers of AAE. It is believed that this task's effectiveness derives from the processing-dependent nature of the task, as opposed to the usual language assessments that are heavily language dependent. We chose six targets for this task, in which the child is asked to repeat a nonsense word two to four syllables long, such as the trial item poo-zle. All six of the actual items were carefully constructed to account for the phonotactics of AAE to ensure that AAE speakers would not be placed at a disadvantage when attempting to repeat them. The nonwords were constructed out of single phonemes (no consonant clusters) and used only those final consonants that in our archive of AAE child speech were least likely to be omitted by AAE speakers. 6,7

We believe that the child's risk status can be easily determined from the response patterns on these 17 diagnostic items. Based on a combined score for all items, the child could be classified as at "the lowest risk for language disorder," "low to medium risk," "medium to high risk," or "the highest risk for disorder." Because this is done in a dialect-neutral way at the outset, it sets the framework for further testing. Typically, in an effort to accommodate divergent dialects such as AAE, speech-language professionals (SLPs) modify standardization guidelines of language tests by adjusting scores or interpreting results in accordance with the child's dialect. Such practices are highly questionable because they violate the tests' standardization protocols. By contrast, our proposed screening items do not differ for $\mathrm{AAE}$ and MAE speakers, and scoring guidelines can be the same for both groups.

What we have chosen to call the Diagnostic Risk Status is indicated by a score that reflects the number of elements indicative of risk. Some responses, like omitting the past tense was are weighted double because they are particular warning signs or "red flags." The higher the score, the greater the risk. The difference between the typically developing and impaired groups in both dialect samples is shown in Figures 3 and 4.

The effects of development and clinical status is significant for all children, although the gap narrows with age, as the significant interaction indicates: Age, $F(5,1002)=56.465$, $p<.0001$; Clinical Status, $F(1,1002)=168.07$, $p<.0001$; Age by Clinical Status, $F(5,1002)=$ $3.172, p=.008$. The gap appears even greater between the clinical groups in the MAE background children: Age, $F(5,362)=23.927$, $p<.0001$; Clinical Status, $F(1,362)=114.95$, $p<.0001$; Age by Clinical Status, $F(5,362)=$ 2.316, $p=.046$.

\section{INTERPRETING PERFORMANCES ON OUR SCREENING ITEMS}

The two scores on our screening items for language variation and diagnostic risk can be interpreted together in forming an overall clinical profile of a child. It is possible to obtain four major performance profiles: MAE/lowest risk; MAE/some risk; some variation from

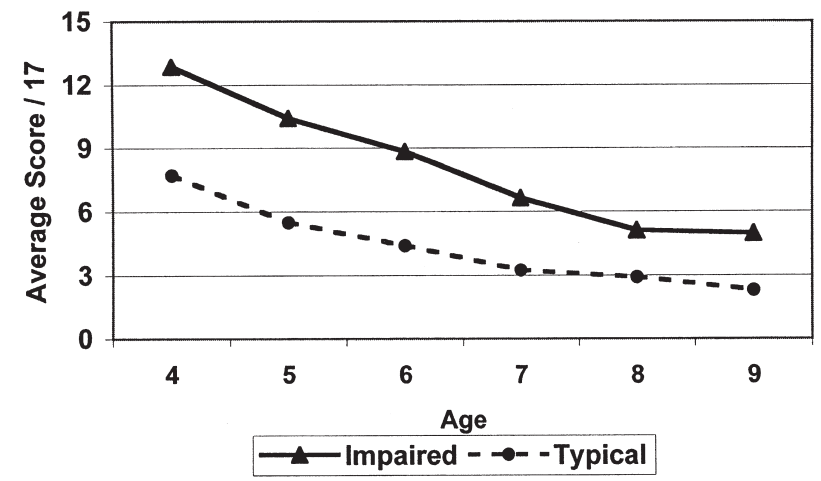

Figure 3 Noncontrastive diagnostic elements by clinical status. 


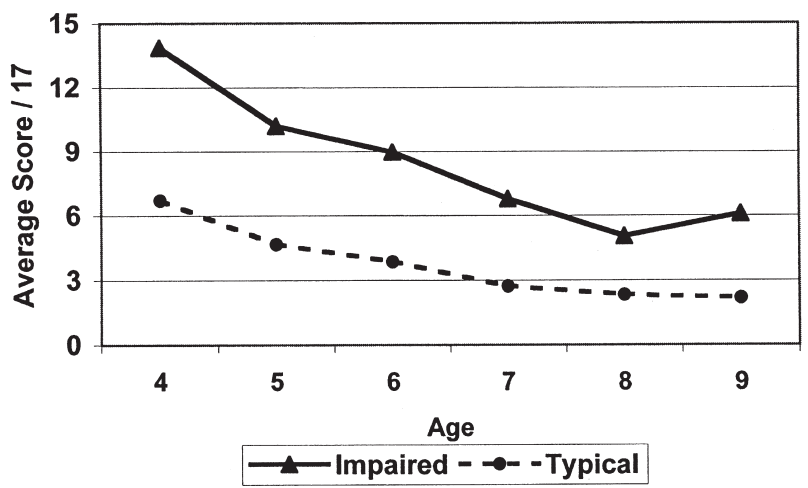

Figure 4 Noncontrastive diagnostic elements by clinical status (Mainstream American English [MAE]-background children only).

MAE/lowest risk; some variation from MAE/ some risk. Each of these profiles is discussed below with interpretative implications.

\section{MAE/Lowest Risk}

The child who falls into this profile has produced all or most of the 15 variation items and all or most of the 17 diagnostic items in a manner consistent with MAE. We believe no further clinical action should be necessary.

\section{MAE/Some Risk}

The child who fits this description is a candidate for further diagnosis to confirm that a language disorder exists and to determine the nature of the problem. He or she has responded to the 15 variation items in the MAE manner, but has done so on relatively few of the 17 diagnostic items. The child's performance on the 15 variation items suggests few difficulties with morphosyntax. Therefore, further diagnosis could focus on other aspects of language along the lines discussed in the articles in this issue (especially Roeper, ${ }^{4}$ P. de Villiers, ${ }^{8} \mathrm{~J}$. de Villiers, ${ }^{9}$ and Seymour ${ }^{10}$ ).

\section{Some Variation from MAE/Lowest Risk}

Children with a certain number of predictable AAE responses on the 15 variation items scored in either the Some or Strong Variation from Mainstream American English category. These children were considered speakers of AAE.
Because many of the AAE responses can be heard in the speech of young children, regardless of dialect, it is possible for non-AAEbackground children to use some of the very patterns that indicate AAE status (e.g., absent third-person $-s$, as in "he talk"). However, we have found that younger children produced enough responses indicative of an AAE pattern that the distinctions between AAE and MAE typical development were observed even at the younger ages.

The important fact about this profile is that AAE children have been identified and not penalized for their use of AAE features. Their performance on the 15 variation items confirmed their status as AAE speakers, but their predicted MAE responses on the 17 diagnostic items showed the lowest risk for a disorder. We believe it is this Some Variation from MAE/ Lowest Risk profile that is most often confused for disorder in other language tests and that an adequate screener must be able to distinguish it from the other the profiles.

\section{Some Variation from MAE/Some Risk}

Of all the profiles this is the most complicated to interpret. The interpretation is clear with respect to the clinical purpose to identify impaired children and not penalize typically developing African American children for speaking AAE. Our screening items appear to achieve this most important objective. The complexity arises from the potential overlap in variation status between AAE children at risk 
and MAE children at risk. The variation status is relatively straightforward when African American children show AAE patterns on the screener and pass the diagnostic items (i.e., they are typically developing AAE speakers), and when typically developing MAE speakers confirm their variation status by their performance on the variation items. However, what is not clear is when children of either dialect show responses consistent with $\mathrm{AAE}$ patterns on the variation items of the screener and also fail the diagnostic items. Because an impaired language system is so unstable, it may show patterns similar to the 15 variation items (deletions such as third-person $/-s /$, do/does, and have/has substitutions and certainly immature phonological patterns such as [f] substituted for $/ \theta /$ ) Consequently, both AAE and MAE language-disordered children may appear to be AAE speakers. In fact, in the field testing sample, more than half of the children from MAE-speaking backgrounds who were identified for and receiving language services spoke with some or strong variation from MAE.

For the child from an MAE background, these problems on the variation items (contrastive features) could be an indication that something is amiss linguistically. But the stronger evidence in either dialect for a language problem will be the child's performance on the diagnostic items (noncontrastive features). As Figure 3 shows, the noncontrastive features distinguish the typically developing from languageimpaired children, but they are an even better diagnostic for MAE background children than for children who speak with strong difference from MAE (Figure 4). This is consistent with the observation made by Seymour ${ }^{11}$ in the introduction to this issue that normal AAE features can appear similar to patterns of language disorder in MAE speakers. The linguistic pattern of an African American child (or a child from another racial or ethnic background) who has a language disorder and comes from a home and community where MAE is the dominant dialectal pattern will mimic those of an AAE speaker. When the features reflect accurate learning of the language model provided to the child, they are markers of dialect. When they are an inaccurate reflection of the child's language model, as in the case of a language-impaired child from an MAE-speaking home, then they generally signal disorder.

Thus, the AAE identifiers work best in preventing children who use AAE patterns and who are typically developing from being misdiagnosed. However, these AAE identifiers, when used as the sole source for dialect identification, can be ambiguous with respect to dialect for children whose system is impaired. Our work attempts to overcome this ambiguity by not relying on precisely those contrastive features for overall assessment. Therefore, when AAE status is suspected for an impaired child based on the variation items, other confirming evidence is necessary, such as family and community background. Some evidence of $\mathrm{AAE}$ or MAE features may be seen in the child's longer responses to other items, particularly in the Pragmatics domain of the proposed comprehensive language test $(\mathrm{P}$. de Villiers $^{8}$ ). Indeed, SLPs know that a single test score is only one piece of the assessment puzzle: To figure out what is going on with a child, it is essential to consider all pieces of information before drawing conclusions that affect a child's future educational plans.

\section{FURTHER DIAGNOSTIC TESTING}

Because our proposed screening items do not constitute a full diagnostic test, children identified as at risk should be given additional testing. For many reasons discussed throughout this issue, the proposed dialect-sensitive language test described in this issue is recommended. But no test replaces the need for ongoing diagnosis. This is especially important for certain aspects of language that have not been examined by the proposed test.

Although criterion-referenced items can form a comprehensive test of language comprising syntax, semantics, pragmatics, and phonology, the one we propose does not directly examine morphology and this aspect is examined only in a limited way in our screening items. The reason for avoiding morphosyntax to this degree is to prevent disadvantage to AAE speakers. However, because morphology has a high probability of being aberrant when a child's language is impaired, it should not be ignored in subsequent stages of diagnosis 
and treatment. Therefore, the following considerations are pertinent to further diagnosis of morphology.

If the child is an MAE speaker who fails our diagnostic screening items and shows difficulty with the variation items by scoring as Some or Strong Variation from MAE, this variation status could be an indication of problems with morphosyntax. There are several options available for testing morphology with MAE-speaking children who give evidence of problems with morphology, and SLPs should follow traditional best practices. ${ }^{12-15}$

In the case of a non-MAE speaker, the objective of assessing morphology would be to determine whether the child's patterns are (1) consistent with noncontrastive targets and (2) typical of AAE speakers in either structure or frequency. Examples of noncontrastive morphological structures would be possessive pronouns (his, hers, ours) past tense copula (It was cloudy), or presentational copula (It's the President). ${ }^{16}$ AAE speakers should not show difficulty with these items unless they have problems with morphology. To determine whether patterns are typical of AAE, there should be an assessment of the frequency of usage. A defining pattern of AAE morphological inflections is optional use, which means they are absent some of the time and present at other times. For example, in the University of Massachusetts archive of AAE child speech, the is copula is typically absent about $20 \%$ of the time. The exact linguistic contexts that govern its presence and absence are not completely known, but a child who is deleting the is copula most of the time is probably showing difficulty.

Most importantly for the African American child, viewing the results of the two types of proposed screening items together clears the "variation due to speech and language dialect" issue out of the way so that if further evaluation is needed, it is done for valid reasons, not superficial ones.

\section{ACKNOWLEDGMENTS}

This work was funded in part by National Institutes of Health (NIDCD) under Contract \# N01 DC8-2104 and Grant \# R01 DC 0217204 to Harry Seymour, Principal Investigator, at the University of Massachusetts Amherst, with Thomas Roeper and Jill de Villiers at the University of Massachusetts and Smith College, as co-investigators. It was accomplished in conjunction with The Psychological Corporation of Harcourt Assessment, Inc., San Antonio, TX.

The tests that are the products of this research collaboration are the Diagnostic Evaluation of Language Variation (DELV) assessments, the DELV Screening Test, DELV Criterion-Referenced edition, and the DELV Norm-Referenced edition. The phrase "evaluating language variation" refers generally to the assessment processes discussed in this issue. The term DELV is the name trademarked by The Psychological Corporation of Harcourt Assessments, Inc., and refers to the specific tests that are the outcome of the extensive research described in this article. The specific tests are referred to as the $D E L V-S T$, or "screener," or the $D E L V-C R, D E L V-N R$, or the "full diagnostic test," as appropriate. Questions about the principles underlying the tests can be referred to the authors of this issue (Seymour, Roeper, de Villiers, de Villiers, Pearson, and Ciolli). Questions about the tests themselves should be addressed to the Project Leader at The Psychological Corporation of Harcourt Assessment, Inc.; Lois Ciolli, Senior Research Director.

\section{REFERENCES}

1. Baugh J. A Re-Examination of the Black English Copula. Austin, TX: Southwest Educational Development Laboratory; 1979

2. Labov W. Contraction, deletion, and inherent variability of the English copula. Language 1969; 45:715-762

3. Wyatt TA. Linguistic Constraints on Copula Production in Black English Child Speech [dissertation]. University of Massachusetts Amherst; 1991

4. Roeper T. Diagnosing language variations: underlying principles for syntactic assessment. Semin Speech Lang 2004;25:41-56

5. Campbell T, Dollaghan C, Needleman H, Janosky J. Reducing bias in language assessment: processing-dependent measures. J Speech Lang Hear Res 1997;40:519-525

6. Abdulkarim L, Bryant $\mathrm{T}$, Seymour HN, Pearson BZ. Consonant clusters in child African 
American English phonology. Poster presented at: the Annual Meeting of the American SpeechLanguage-Hearing Association, San Francisco, November 18-21, 1999

7. Abdulkarim L, Bryant $\mathrm{T}$, Seymour $\mathrm{HN}$, Pearson BZ. Final consonants in child African American English phonology. Poster presented at: the Annual Meeting of the American SpeechLanguage-Hearing Association, San Francisco, November 18-21, 1999

8. de Villiers PA. Assessing pragmatic skills in elicited production. Semin Speech Lang 2004;25:xxx-xxx

9. de Villiers JG. Cultural and linguistic fairness in the assessment of semantics. Semin Speech Lang 2004;25: $x x x-x x x$

10. Pearson BZ, Ciolli L. Distinguishing dialect and development from disorder: case studies. Semin Speech Lang 2004;25:xxx-xxx

11. Seymour HN. The challenge of language assessment for African American English-speaking children: a historical perspective. Semin Speech Lang 2004;25:3-12

12. Muma JR. Language Handbook: Concepts, Assessment, Intervention. Englewood Cliffs, NJ: Prentice Hall; 1978

13. Bloom L, Lahey M. Language Development and Language Disorders. New York: John Wiley; 1978

14. Nelson N. Childhood Language Disorders in Context: Infancy through Adolescence. Columbus, OH: Merrill; 1993

15. Paul R. Language Disorders from Infancy through Adolescence: Assessment and Interventions, 2nd ed. St. Louis, MO: Elsevier Science; 2001

16. Benedicto E, Abdulkarim L, Garrett D, Johnson V, Seymour H. Overt copulas in African American English speaking children. In: Greenhill A, Hughes M, Littlefield H, Walsh H, eds. Proceedings of the 22nd Annual Boston University Conference on Language Development. Somerville, MA: Cascadilla Press; 1998:50-57 